\title{
BRITISH FOOD MANUFACTURING INDUSTRIES RESEARCH ASSOCIATION
}

\section{OPEN DAY}

$\mathrm{T}$ HE fine modern laboratories of the British Food Manufacturing Industries Research Association at Leatherhead, Surrey, were occupied in the latter part of 1950, and the opening ceremony performed in November of the following year was then made the occasion of an open day ${ }^{1}$. On June 21 last the laboratories were once again thrown open to representatives of member firms, while representatives of other research organizations, of the scientific, technical, trade and national Press, and others were present by invitation. Some three or four hundred visitors saw exhibits illustrating the course of research during the past four years and the main aspects of the current research programme.

The interests of the Research Association cover a wide range of manufactured foods, with the exception of flour-milling, bread and bakery products, dairy products, and fruit and vegetable canning, all of which are the concern of separate research organizations. The research programme may be divided into three main categories: analytical problems in the routine control of quality; problems directly concerned with particular aspects of food manufacture; and more general investigations into fundamental principles with a bearing on the quality of the manufactured foods concerned. Some aspects of the work having wider scientific interest are outlined below.

The rheological properties of certain foods, including such diverse products as toffee, margarine, chocolate (in its fluid state), and starch, gelatine, agar and pectin jellies, have received considerable attention. A number of experimental pieces of apparatus were displayed, each designed to measure the force required to deform, or cause flow in, margarine and its allied product, compound cooking fat. One of these in particular, the 'extrusion plastometer', shows much promise in relating plasticity and 'spreadability' to readings taken on the instrument. Chocolate is processed during manufacture in a liquid state, and measurement of its rheological properties is of great importance in the chocolate industry. It has been shown to behave as a Bingham material, and measurement of its 'viscosity' raises considerable difficulty in factory practice. A number of commercial and experimental instruments which have shown promise of proving useful in factory control were exhibited. Investigations into the pasting of starch have been carried out by means of the corn industries viscometer. The measurement of the properties of jellies of various types is of interest in a number of foodstuffs. The Research Association has recently contributed largely to the design of the F.I.R.A. jelly tester, which incorporates several refinements, particularly in respect of design of the spade and reduction of friction, in comparison with the older B.A.R. tester. Recent work on the gelling properties of agar has shown how the instrument may be used for characterizing jellies and obtaining information beyond tho measurement of pure 'jelly strength'.

The Research Association is studying the plastic composition and crystallization behaviour of cocoa butter and other fats through determinations of the specific heats and heat contents over a wide range of temperatures. From the measurements obtained, the percentage of liquid phase at a given temperature may be calculated. An adiabatic calorimeter has been constructed for the purpose of these studies, a description of which will shortly be published elsewhere by Mr. E. H. Steiner. The procedure followed is intermediate between that described by A. E. Bailey et al. ${ }^{2}$ and that of J. Straub and R. N. M. A. Malotaux as adapted by S. V. Vaeck ${ }^{8}$. The investigation is important in the study of the tempering of chocolate and the use of alternative fats in certain types of confectionery. The control of particle size is of importance in many food products, such as icing sugar, cocoa powder, chocolate and starch. Among the exhibits were a commercial vortex sieve for cocoa powder, a modified Andreasen sedimentation apparatus for ehocolate and a Lea and Nurse air-permeability apparatus for the determination of specific surface of several materials.

In the field of biochemistry, a study of the nature of the changes undergone by meat products, particularly colour changes in sausages, ham and other cured meats, is being made from a fundamental point of view ; a note on one aspect of this work was recently published by J. I. Anderton and D. J. Locke $e^{4}$. The separation and study of vitamin $A$ and related substances in liver oils is being undertaken by the use of a 'short-path' molecular still, chromatographic methods and the use of the quartz spectrophotometer. Fruits for jam-making and brined vegetables for the manufacture of pickles are both subject to undesirable tissue breakdown under certain conditions. The part played by pectolytic enzymes of the fruits and vegetables and those produced by moulds naturally present on fresh fruit and vegetables is being investigated in this respect. The Research Association maintains an extensive mierobiological laboratory for the study of both the desirable organisms that are necessary to such processes as the curing of bacon and the preparation of pickles, and the spoilage organisms which are a source of trouble in canned meat and fish products, and in pickles and sauces. Investigations of the thormal death-rate characteristics of mould spores which may cause spoilage in jam and of the bacteria and yeasts responsible for spoilage in pickles are being carried out.

On the technological level, the Research Association maintains pilot plant for studying the rate of heat penetration into canned goods under commercial conditions, and a laboratory-sized apparatus for carrying out pasteurization tests on jars of pickled onions has recently been designed and constructed. The packaging of manufactured foods is an integral part of the interests of the Research Association. While it does not concem itself with the study of the properties of the various wrapping and packaging materials, it is concerned with the choice of material best suited to particular products. In this connexion, a number of studies are undertaken, such as measurement of the vapour pressure of confectionery products, the protection of metal closures on glass packages from any corrosive effects due to the nature of the product packed in them or, conversely, discoloration of the product in contact with the closure, and the effects of transparency and 
air-permeability of wrappers for margarine. The permeability of rubber sealing rings to gases and volatile substances is a cause of deterioration in meat and fish pastes, and the most desirable formulation for such rubber rings is being sought in collaboration with the manufacturers.

1 See Nature, 168, 1108 (1951).

2 Oil and Soap, 21, 293 (1944).

${ }^{3}$ Int. Choc. Rev., 6, 350 (1951).

4 Nature, 175, 818 (1955).

\section{RADIATION CHEMISTRY OF SOLUTIONS}

$\mathrm{T}$ HE fifth annual meeting of the Société de Chimie Physique was held in Paris during May 31-June 3, the subject of the meeting being the chemical effects of ionizing radiation. It was organized by Prof. G. Emschwiller and was attended by chemists and physicists from Europe, the United States and the U.S.S.R.

Radiation chemistry is complicated by the fact that the primary events are confined to the tracks of the ionizing particles. It was pointed out that in aqueous solutions the yield varies with radiation quality, the principal change occurring in the range 6.5-25 keV./ $\mu$ mean linear energy transfer of the radiation. On the assumption that only those parts of the ionizing tracks where the linear energy transfer is greater than $25 \mathrm{keV} . / \mu$ are effective, and taking $\delta$-rays into account, a curve for the efficiency of different radiations has been calculated which is in good agreement with the experimental data for the molecular hydrogen yield (L. H. Gray). A tentative mathematical picture was given of the kinetics of radicals formed in a track (J. L. Magee). With increasing intensity, the tracks overlap and the kinetics of homogeneous solutions apply. This change was demonstrated using diphenylpicryl bydrazine in chloroform (A. Chapiro). There is a possibility that excited water molecules play a part in the indirect effects in dilute solutions (J. Weiss). However, the strict proportionality of fluorescence and sodium salicylate concentration in an aqueous solution exposed to $\alpha$-radiation suggests the absence of indirect excitation of the solute (N. Miller).

The most useful system for the chemical measurement of dose is still a solution of $0.001 M$ ferrous ions in $0 \cdot 1 M$ sulphuric acid. Several recent determinations for $\mathrm{X}$-rays and $\beta$-rays have all favoured a value close to $G_{\mathrm{Fe}^{+++}}=15.5$ molecules per $100 \mathrm{eV}$. (N. Miller ; A. J. Swallow ; M. Cottin). An accurate determination has been carried out with $2 \mathrm{MeV}$. electrons and gives $G_{\mathrm{Fe}^{+++}}=\mathbf{1 5} \cdot 45 \pm 0.11$ (R. H. Schuler and A. O. Allen, reported by N. Miller). The kinetics for the oxidation of $10^{-3} M$ ferrous ions in $0 \cdot 1 M$ sulphuric acid are fairly well understood, but are totally inadequate to explain the high yield $\mathrm{GFe}_{\mathrm{Fe}^{+++}}=63$ obtained in aerated solutions of $2 \times 10^{-2} M$ ferrous ions in $4 M$ sulphuric acid (Proskurnin, quoted by S. S. Medvedev). The sensitivity of yield to the presence of impurities was demonstrated with the $\mathrm{Fe}_{\Theta}++$ system (N. Miller ; C. Vermeil). For the measurement of exceptionally high doses, oxalic acid was recommended (I. Draganic). The measurement of dose by the ionization method depends upon the energy $W$ required to form one ion-pair in air. Recent measurements with polonium $\alpha$-rays give values close to $W=\mathbf{3 5 . 5} \mathrm{eV}$., in agreement with older data. Now results for fast electrons obtained from a comparison of absolute countingrates and ionization measurements of sodium-24, cobalt-60, gold-198, iodine-131 and phosphorus-32 lead to a value of $W=34 \pm 1 \mathrm{eV}$. in air, differing slightly from $W=32.5 \mathrm{eV}$., the value widely used in radiological work (W. Emery, reported by L. H. Gray).

It was generally agreed that the initial products in the radiolysis of water are the free hydrogen and hydroxyl radicals and the molecular products hydrogen and hydrogen peroxide. The mechanism giving rise to the formation of the molecular products is not understood, but evidently it occurs in those parts of the tracks where the linear energy transfer is very high. The yields of molecular hydrogen are less affected than the yields of hydrogen peroxide by the presence of various solutes (M. Lefort and $\mathbf{M}$. Cottin). New light was thrown on these processes by studies of radiation-induced polymerization and of the production of hydrogen peroxide in gas-free aqueous solutions of acrylonitrile. Both the monomer and polymer are inert to hydrogen peroxide, but readily combine with hydrogen and hydroxyl radicals. The yield of hydrogen peroxide varies with monomer concentration. It is at a maximum at $10^{-2} M$ and drops by a factor of 6 at the highest monomer concentration used, $0.5 M$, when the monomer reacts with precursors of hydrogen peroxide even in the densest parts of the tracks (E. Collinson, F. S. Dainton and G. F. MeNaughton). No similar demonstration of precursors of molecular hydrogen has yet been made. It might be formed directly from one water molecule, but the dependence of the yield on the linear energy transfer suggests a recombination process (M. Haĩssinsky; G. Stein; M. Magat). Oxygen-18 has been used in a study of the production and decomposition of hydrogen peroxide by radiation (E. J. Hart, S. Gordon and D. A. Hutchinson).

Some of the aqueous solutions investigated are of biochemical interest. Irradiation of phosphoric acid esters of the homologous series of methyl to amyl alcohols shows that the larger molecules are more stable. The main products of decomposition are inorganic phosphate, acyl phosphate, aldehyde and hydrogen peroxide. All solutions show an aftereffect if irradiated in the presence of oxygen. Analogous results with solutions of mononucleotides were reported (R. W. Wilkinson and T. F. Williams; G. Scholes; M. Daniels). Radiation effects on catalase in dilute aqueous solution show that quantitative results depend to a very large extent on the purity of the enzyme preparation. The enzyme is inactivated by hydroxyl radicals and not by hydrogen radicals as has been previously assumed (W. M. Dale and C. Russell; P. Bonet-Maury and F. Patti; H. C. Sutton, quoted by L. H. Gray). In contrast, reduction processes occur in solutions of methylene blue, coenzyme I and diphosphopyridine nucleotide (G. Stein ; A. J. Swallow).

Irradiation of aqueous solutions of polyvinylpyrrolidine and other water-soluble polymers leads to degradation if the concentration is less than 0.3 per cent, and to cross-linking if more than 1 per cent (A. Charlesby and P. A. Alexander). The mechanism of cross-linking, however, is not yet understood (A. Henglein; Y. Landler). Irradiation of air-free solutions of methacrylic acid leads to polymerization which continues after the end of the exposure. The resulting polymer, dried and exposed to air for several hours, can still initiate polymerization in new unirradiated monomer solutions (M. Fox and P. A. 